www.jmscr.igmpublication.org

Index Copernicus Value: 79.54

ISSN (e)-2347-176x ISSN (p) 2455-0450

crossref DOI: https://dx.doi.org/10.18535/jmscr/v7i5.81

Journal Of Medical Science And Clinical Research

IGM Publication

An Official Publication of IGM Publication

\title{
Evaluation of Cardiovascular Dysfunction among non alcoholic Fatty Liver Disease Patients
}

\author{
Authors \\ Dr Parimal Sarkar ${ }^{1 *}$, Dr Subhadip Paul ${ }^{2}$ \\ ${ }^{1}$ Assistant Professor, Agartala Govt. Medical College \\ ${ }^{2}$ Post Graduate Trainee, Agartala Govt. Medical College, \\ *Corresponding Author \\ Dr Parimal Sarkar \\ Assistant Professor Agartala Govt. Medical College
}

\begin{abstract}
Introduction: Nonalcoholic fatty liver disease has become the most common liver disease worldwide. The prevalence of NAFLD is rising rapidly because of ongoing epidemics of obesity and type 2 diabetes. NAFLD is also an emerging problem in the Asia Pacific region, where it is likely to increase in the future. The estimated prevalence of NAFLD is currently $25 \%$ of the global adult population. The prevalence in the morbidly obese population has been estimated around 75-92\%.The presence of cardiovascular dysfunction in liver cirrhosis has been described, which is usually a dilated cardiomyopathy with decreased ejection fraction. Only in the last 2 decades an increasing body of evidence shows that NAFLD has to be considered as a significant independent risk factor for cardiovascular disease. Long term follow-up studies demonstrate cardiovascular mortality to be the most important cause of death in NAFLD patients.

Aims of the Study: To study the type of cardiac dysfunction among nonalcoholic fatty liver disease patients. To study the association between cardiac dysfunction with stages of cirrhosis.

Materials and Method: It was a cross sectional study done on 40 patients admitted in medicine department of AGMC \& GBP Hospital, Agartala between April.2018 to Nov.2018.

Results: Out of all patients of NAFLD $60 \%$ were females and $40 \%$ were males. So female preponderance is found in development of NAFLD. Out of all patients of NAFLD 55\% were from rural areas and $45 \%$ were from urban areas. Out of all patients $40 \%$ patients had normal ecg, $17.5 \%$ patients had RBBB, $12.5 \%$ had $1^{\text {st }}$ degree heart block and Bifascicular block respectively, $10 \%$ had $L B B B, 7.5 \%$ had $2^{\text {nd }}$ degree heart block.Out of all patients with CTP score A had grade 1 diastolic dysfunction in $10.5 \%$ patients. Patients with CTP score B had grade 1 diastolic dysfunction in 52.6\% patients, grade 2 diastolic dysfunction in 10\% patients. Patients with CTP score C had grade 2 diastolic dysfunction in $90 \%$ patients. So this study shows that decompensated cirrhosis with CTP score C had more grade 2 diastolic dysfunction compare to CTP score B. Out of all patients with CTP score A had pericardial effusion in $4 \%$ patients. Patients with CTP score $B$ had pericardial effusion in $48 \%$ patients. Patients with CTP score C had pericardial effusion in $48 \%$ patients. So this study shows that decompensated cirrhosis with CTP score B \& C had more pericardial effusion compare to CTP score A.

Discussion: This study shows that females are more prone to develop Non Alcoholic Fatty Liver Disease (NAFLD), peoples from rural areas are more affected than urban areas. Chi-Square test shows strong association between CTP Score with pericardial effusion with a p value of 0.014(<0.05). Chi-Square test shows strong association between CTP Score with diastolic dysfunction with a p value of $0.001(<0.05)$. The study reflects that ecg and echocardiography testing should be an important component of evaluation cardiovascular dysfunction among nonalcoholic fatty liver disease. This study will help in reducing cardiovascular related morbidity and mortality among nonalcoholic fatty liver disease patients. Abbreviations: NAFLD: Non alcoholic fatty liver disease, RBBB: Right bundle branch block, LBBB: Left bundle branch block, CTP: Child-turcotte-pugh, CVD-Cardiovascular disease.
\end{abstract}




\section{Introduction}

Non-alcoholic fatty liver disease (NAFLD) has become a major cause of chronic liver disease world wide and $25 \%$ of the global adult population is potentially affected by the disease. A total of $3 \%$ to $10 \%$ of all children and about $34 \%$ of children who are obese in developed countries are thought to have NAFLD. The increasing prevalence of these diseases is related to unhealthy lifestyles, particularly unhealthy diet, which increases NAFLD. NAFLD are often found those who are obese or have features of poor metabolic health even at normal weight. NAFLD is relatively under diagnosed and long-term outcomes of hepatic and extrahepatic manifestations of NAFLD are compromised.

Liver plays a crucial role in lipid and glucose homeostasis and stays in the center of cardiometabolic disease. Thus imbalance in calorie-intake and expenditure leads into deposition of fat in the liver. NAFLD is part of and victim of metabolic derangement and in the emergence of cardiovascular disease, type 2 diabetes, and chronic kidney disease. Compared with healthy individuals, persons with NAFLD had a 1.5 -6 times higher risk of cardiovascular disease, diabetes.

NAFLD can be categorised histologically into non-alcoholic fatty liver and non-alcoholic steatohepatitis (NASH). Non-alcoholic fatty liver is defined as the presence of atleast 5\% hepatic steatosis without evidence of hepatocellular injury. NASH is defined as the presence of a least $5 \%$ hepatic steatosis and inflammation with hepatocyte injury with or without fibrosis.

Cardiovascular disease is the main cause of death in NAFLD patients. Patients with NAFLD exhibit endothelial dysfunction of conducting vessels, stiffness of arterioles and carotid intimal media thickness. Endothelial dysfunction of the systemic circulation is mainly due to asymmetric dimethylarginine (ADMA) is an endogenous antagonist of nitric oxide synthase, positively linked with CVD. Other markers for endothelial dysfunction is endocan. Beside that the level of circulating endothelial progenitor cells is also reduced in NAFLD, which has endothelial repair activity.

The liver is the main organ handling amino acids including homocysteine. Elevated serum homocysteine is frequently reported in NAFLD. Homocysteine causes disruption of the intrahepatic vasculature with impaired nitric oxide formation. Hepatic microvasculature shows distortion of the sinusoidal pattern, which leads into increase in portal pressure.

Vascular endothelial growth factor (VEGF), plasminogen inhibitor activator 1 level is increased in NAFLD patients, which has an active role in atherogenesis and plaque formation. Factor VII, von willebrand factor level is also increased and antithrombin III is decreased. NAFLD patients are in thrombotic state resulting in increase in blood viscosity with increased cardiovascular disease. Increased circulating markers IL-6, TNF alpha,CCL3, sICAM-1,hsCRP in NAFLD related to cardiovascular disease. Fetuin-A (FetA) is a hepatokine has positive link between NAFLD and cardiovascular disease. Adipokines are implicated in NAFLD and CVD.

\section{Materials and Method}

It was a cross sectional study done on 40 patients admitted in medicine department of AGMC \& GBP Hospital, Agartala between April.2018 to Nov.2018.

\section{Inclusion Criteria}

- Adult patients with cirrhosis of liver and no significant alcohol consumption (defined as greater than 40 grams per day).

\section{Exclusion Criteria}

- Patients with HBV, HCV positivity.

- Liver diseases of other known causes.

- Patients consuming significant amount of alcohol or druds such as ATT, estrogen, methotrexate, tamoxifen, amiodarone.

- Pregnant women.

- Unwiling or incapacity to provide informed consent. 


\section{Method of Data Collection}

Patients who has undergone USG of whole abdomen examination in the Radiology department for some or other reason with no history of alcohol intake. Cardiovascular function was evaluated with ECG and echocardiography.

\section{Operational definitions}

Non-Alcoholic Fatty Liver Disease (NAFLD)

NAFLD is defined as the presence of fat in the liver either on imaging or on liver histology after the exclusion of secondary causes of fat accumulation in the liver.

\section{Non-Alcoholic Steato Hepatitis (NASH)}

When NAFLD is associated with raised transaminase levels, the diagnosis of NASH is made (Normal AST 12-38 U/L and ALT 7-41U/L

\section{Statistical analysis}

The collected data has been summarized in terms of tables and figures. The arithmetic mean and standard deviation has been calculated. Chi square test has been used to find association between two categorical variables. A p value of $<0.05$ has been considered statistically significant. Data analysis was done using SPSS version 20.
A total of 40 patients were worked up according to the procedure detailed in the methodology and the data obtained thereby was presented and analysed.

\section{Gender Wise Distribution}

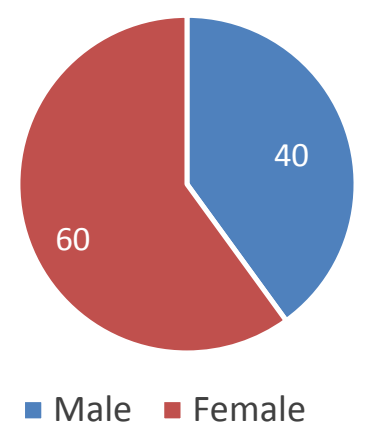

\section{Urban vs Rural Population}

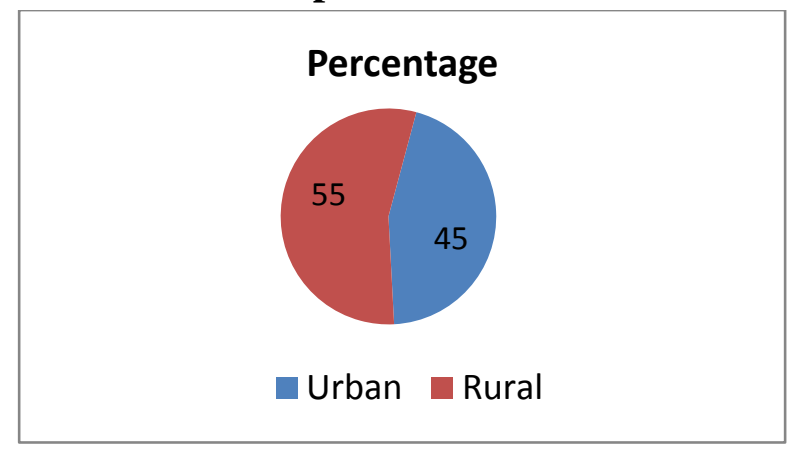

\section{ECG Abnormalities in NAFLD}

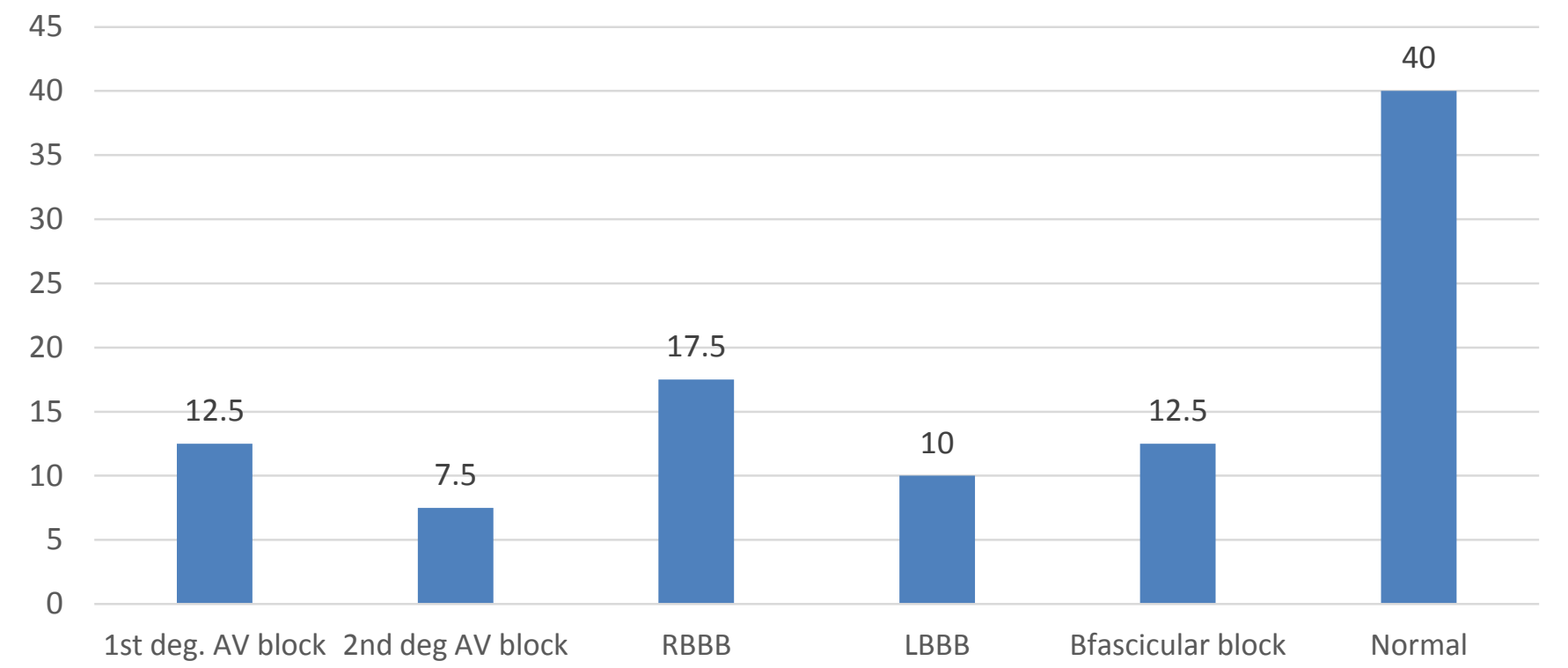




\section{JMSCR Vol||07||Issue||05||Page 500-504||May}

Diastolic Dysfunction in different stages of CTP

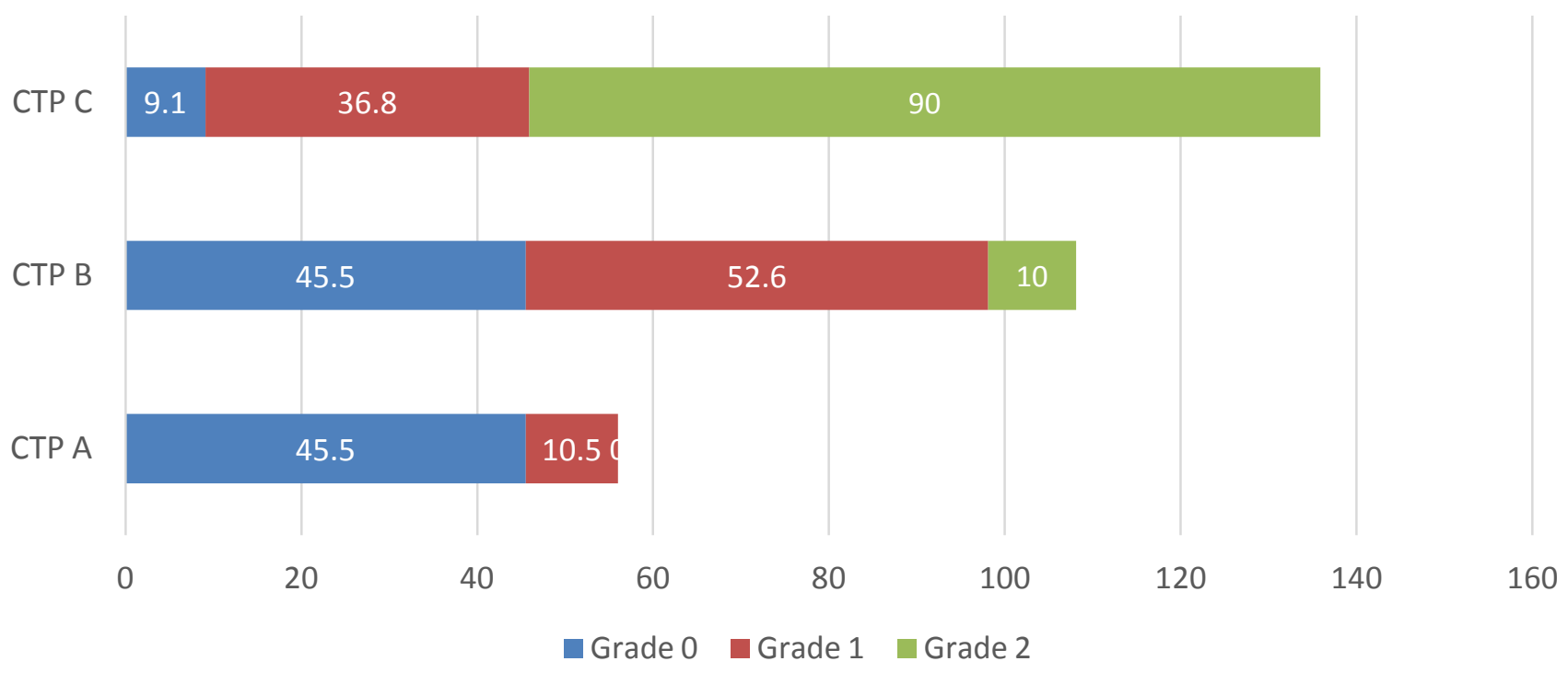

Pericardial effusion in stages of CTP

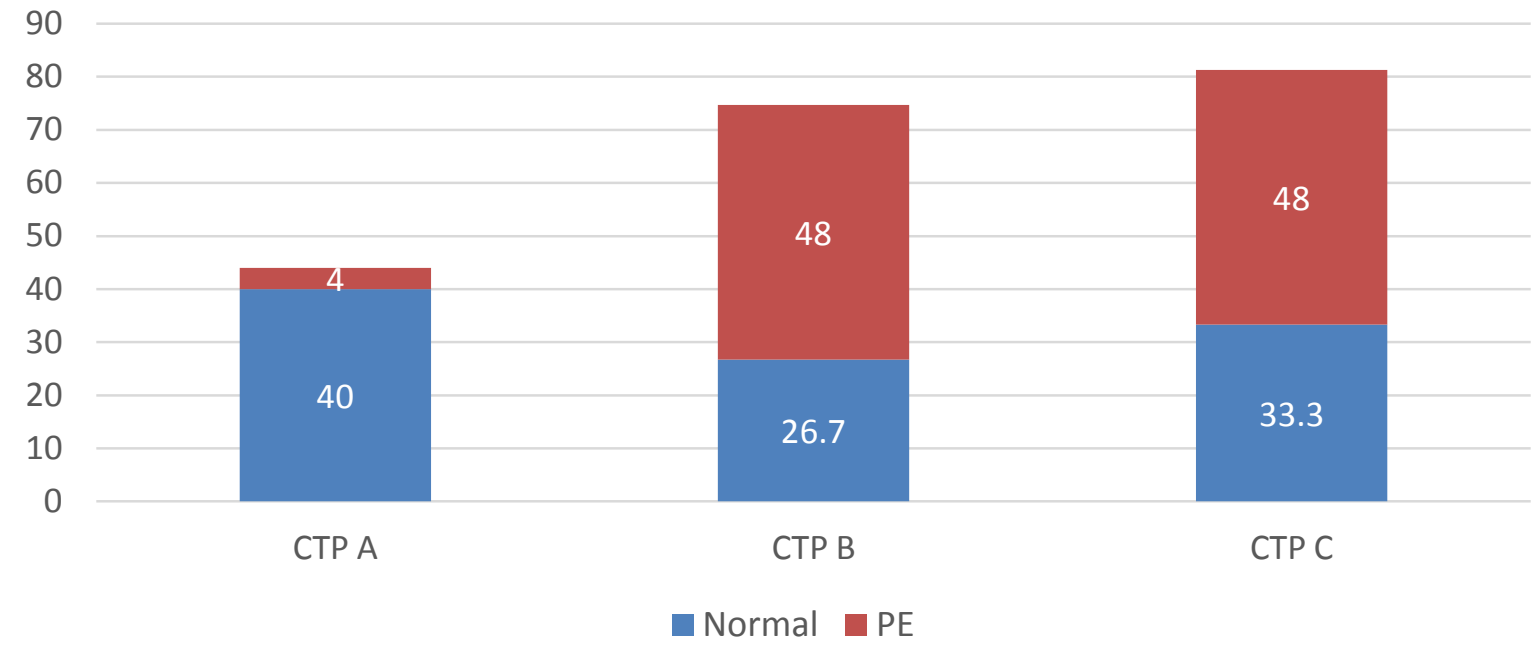

Frequency of grades of HE in NAFLD

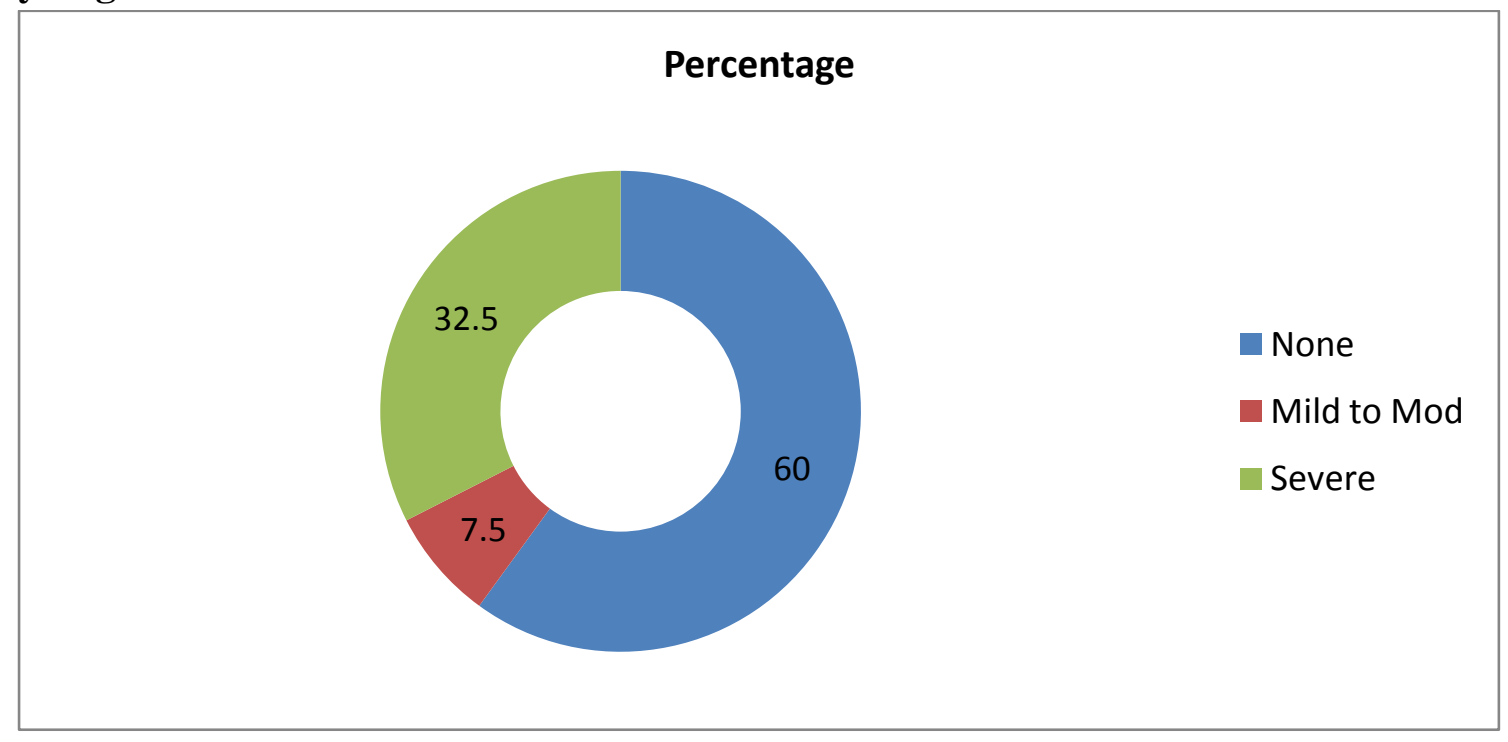




\section{Discussion}

Out of all patients of NAFLD $60 \%$ were females and $40 \%$ were males. So female preponderance is found in development of NAFLD. Out of all patients of NAFLD 55\% were from rural areas and $45 \%$ were from urban areas. Out of all patients $40 \%$ patients had normal ECG, $17.5 \%$ patients had RBBB, $12.5 \%$ had $1^{\text {st }}$ degree heart block and Bifascicular block respectively, $10 \%$ had LBBB, $7.5 \%$ had $2^{\text {nd }}$ degree heart block. Out of all NAFLD patients with CTP score A had grade 1 diastolic dysfunction in $10.5 \%$ patients. Patients with CTP score B had grade 1 diastolic dysfunction in $52.6 \%$ patients, grade 2 diastolic dysfunction in $10 \%$ patients. Patients with CTP score $\mathrm{C}$ had grade 2 diastolic dysfunction in $90 \%$ patients. So this study shows that decompensated cirrhosis with CTP score C had more grade 2 diastolic dysfunction compare to CTP score B. Out of all NAFLD patients with CTP score A had pericardial effusion in $4 \%$ patients. Patients with CTP score B had pericardial effusion in $48 \%$ patients. Patients with CTP score C had pericardial effusion in $48 \%$ patients. So this study shows that decompensated cirrhosis with CTP score B \& C had more pericardial effusion compare to CTP score A. This study shows that females are more prone to develop Non Alcoholic Fatty Liver Disease (NAFLD), peoples from rural areas are more affected than urban areas. ChiSquare test shows strong association between CTP Score with pericardial effusion with a $\mathrm{p}$ value of $0.014(<0.05)$. Chi-Square test shows strong association between CTP Score with diastolic dysfunction with a $\mathrm{p}$ value of $0.001(<0.05)$. The study reflects that ECG and echocardiography testing should be an important component of evaluation cardiovascular dysfunction among nonalcoholic fatty liver disease patients. This study will help in reducing cardiovascular related morbidity and mortality among nonalcoholic fatty liver disease patients.

\section{References}

1. Charlton MR, Burns JM, Pedersen RA, Watt KD, Heimbach JK, Dierkhising RA. Frequency and outcomes of liver transplantation for nonalcoholic steatohepatitisin the United States. Gastroenterology 2011;141:1249-1253 .j.gastro.2011.06.061.

2. Said A, Gagovic V, Malecki K, Givens ML, Nieto FJ. Primary care practitioners survey of non-alcoholic fatty liver disease. Ann Hepatol 2013;12:758-765.

3. Bergqvist C-J, Skoien R, Horsfall L, Clouston AD, Jonsson JR, Powell EE. Awareness and opinions of non-alcoholic fatty liver disease by hospital specialists. Intern Med J 2013;43:247-253. org/10.1111/j.1445-5994.2012.02848.x.

4. Ekstedt M, Hagström $\mathrm{H}$, Nasr $\mathrm{P}$, Fredrikson M, Stål P, Kechagias S, et al. Fibrosis stage is the strongest predictor for disease-specific mortality in NAFLD after up to 33 years of follow-up. Hepatology 2015;61:15471554.org/10.1002/hep. 27368.

5. Satapathy SK, Sanyal AJ. Epidemiology and natural history of non-alcoholic fatty liver disease. Semin Liver Dis 2015; 35:221-235. org/10.1055/s-0035-1562943.

6. Anderson EL, Howe LD, Jones HE, Higgins JPT, Lawlor DA, Fraser A. The prevalence of non-alcoholic fatty liver disease in children and adolescents: a systematic review and meta-analysis. PLoS One 2015; 10: e0140908.3 Alisi A, Feldstein AE, Villani A, Raponi M. 